\title{
PROCESOS EDUCATIVOS EN EDUCACIÓN INFANTIL MEDIANTE EL GRAFISMO Y LA ESCRITURA
}

\section{Educational processes in Early Childhood Education through graphic design and writing}

\author{
María Luisa Benítez SÁNCHEZ* y Cristina SÁNCHEZ ROMERO** \\ * Escuela internacional de doctorado UNED \\ Correo-e: arlexmadrid1992@telefonica.net \\ ** UNED. Facultad de Educación \\ Correo-e: csanchez@edu.uned.es
}

Recibido: 10/11/2018; Aceptado: 4/12/2018; Publicado: 30/12/2018

Ref. Bibl. MARÍA LUISA BENÍTEZ SÁNCHEZ y CRISTINA SÁNCHEZ ROMERO. Procesos educativos en Educación Infantil mediante el grafismo y la escritura. Enseñanza \& Teaching, 36, 2-2018, 195-214.

RESUMEN: Esta investigación aborda la educación del grafismo y la escritura con un enfoque motriz, en lugar del visual utilizado tradicionalmente, desarrollando movimientos y desplazamientos para aprehender sentido y dirección y plasmarlos en trazo. La muestra se compone $(\mathrm{N}=113)$ de niños de Educación Infantil de 5 años pertenecientes a cinco centros públicos con contextos socioculturales distintos. Se ha seguido una metodología empírica apoyada en la observación participante junto con instrumentos como los materiales empíricos para medir el nivel gráfico de los alumnos y la entrevista a los tutores. Los resultados muestran que los alumnos se inician en la escritura occidental de manera eficaz y creativa a través del movimiento vivenciado que facilita su aprendizaje, mejora las posibilidades motrices, el conocimiento correcto del espacio, la trayectoria del trazado y la ejecución del grafismo, permitiendo iniciar a los alumnos en la escritura occidental de manera eficaz y creativa. Entre las conclusiones destacamos que educar el gesto gráfico desde el movimiento motriz es una alternativa potente en Educación Infantil porque facilita la resolución del problema de aprendizaje del trazado y la escritura. Los resultados 
muestran que al ser una manera de enseñar más lúdica, dinámica y creativa que el sistema tradicional y al ser el niño protagonista, la asume como suya. De esta manera se promueve la ayuda y la valoración positiva para la adquisición de competencias que conducen a aprender a aprender.

Palabras clave: trazado; escritura; movimiento vivenciado; motricidad; Educación Infantil.

SUMMARY: This research shows how developing handstroke and writing through experiential movement facilitates learning and improves motor skills, spatial awareness, the trajectory of the stroke and the execution of handwriting. The sample for this study is made up of 113 early years children (most of them 5 years old) from 5 different public schools and socio-cultural backgrounds. The evaluation techniques used consisted of a test to measure the subjects' writing level and a questionnaire for their teachers. The results show that students become more familiarised with the intricacies of western handwriting through experienced movement and creative learning. Among the conclusions we emphasize that educating the graphic gesture from the motive movement is a powerful alternative in Infantile Education because it facilitates the resolution of the learning problem of the layout and the writing; The results show that being a way to teach more playful, dynamic and creative than the traditional system and being the protagonist child, he assumes it as his own. In this way, help and positive assessment is promoted for the acquisition of skills that lead to learning to learn.

Key words: stroke; handwriting; experienced movement; motor skills; Primary education.

\section{INTRODUCCIÓN}

La necesidad de comunicación del ser humano ha determinado un hecho diferencial con las demás especies; su capacidad para crear instrumentos para expresarse y comunicarse como el lenguaje, convirtiéndose en un ser social. El niño pequeño, activo y curioso por naturaleza, cubre su necesidad de comunicación cuando puede intervenir en el entorno, expresarse, crear caminos para salir de sí mismo y adquirir habilidades para transmitir y comunicar sus ideas, sentimientos, deseos... En esa tarea se sirve del lenguaje, de los diversos lenguajes, que son "los medios que nos permiten comunicarnos con nosotros mismos y los demás, con la naturaleza, la sociedad y la cultura» (Marín, 1984: 22). Pero el niño también utiliza los lenguajes para entender lo que le rodea, manejando para comunicarse tres sistemas de representación: el movimiento, el dibujo o la imagen y la lengua, y el desarrollo supone dominar progresivamente los tres (Bruner, 1984).

Al utilizar distintos lenguajes la mente crea imágenes sonoras, icónicas o dinámicas con sus elementos estéticos, sentimentales y emocionales; realiza distintas operaciones mentales; utiliza sistemas diversos de expresión y comunicación con 
sus formatos específicos, y ensaya experiencias perceptivas nuevas, generando nuevos conocimientos y produciendo enriquecimiento cognitivo.

Los medios de expresión, por su parte, adquieren mayor riqueza cuando actúan con sus propios elementos, renovándose por contacto entre ellos (Arnheim, 1989), así la escritura y la expresión pictórica han ido desarrollándose la una a partir de la otra.

La lengua, entendida por algunas escuelas lingüísticas como la capacidad humana que da forma al pensamiento y el conocimiento, es una herramienta constructora de ideas y conocimientos a lo largo de la vida, contribuye a la socialización y el desarrollo del individuo y es determinante en la adquisición del conocimiento y la cultura de los pueblos. Como función, es capacidad de expresión y comunicación como producto, es un sistema de signos, términos y expresiones cuyo ámbito de conocimiento es el entorno social y, como habilidad, necesita para su desarrollo la interacción con otras personas para obtener conocimientos de la lengua oral y escrita de la sociedad en que se vive.

El lenguaje permite al niño expresarse, dominar y organizar su entorno, es determinante en su desarrollo integral, el instrumento más importante de desarrollo cognitivo y, según Bruner (1984), el medio imprescindible para procesar las percepciones y llegar al conocimiento. Pero no es suficiente para explicar el pensamiento, porque las estructuras que lo identifican nacen de la acción y sus mecanismos sensoriomotores que son más profundos que los lingüísticos (Piaget, 1985). Los estudios epistemológicos de Piaget muestran que solo a partir de la experiencia vivenciada con participación activa de la motricidad se pueden elaborar estructuras fundamentales de pensamiento abstracto, y como en la vivencia... «lo intelectual y lo afectivo van íntimamente unidos». (Lapierre y Aucouturier, 1985: 9), la expresión motriz vivenciada es esencial para el desarrollo integral.

En sus primeros años de vida el niño utiliza el sistema sensitivo y la actividad para captar el mundo, va aprende con y a través del cuerpo en movimiento, «un instrumento directo de conocimiento, aprendizaje y educación integral» (Calmy, 1977: 9). Aprende desde el movimiento, pero con connotaciones psicológicas que superan lo puramente biomecánico (Arnaiz y Bolarín, 2016). Primero conoce su cuerpo, posibilidades y limitaciones y puede explorar, adquirir aprendizajes básicos, realizar experiencias en su entorno físico y social y adquirir autonomía. En el desarrollo psíquico intelectivo y emocional también interviene el movimiento, porque «el desarrollo representa un recorrido desde el movimiento y la acción a la representación y el pensamiento» (Hernández, 2015: 16) y «el paso de la reacción motriz espontánea a la organización perceptivo-motriz consciente parece ser el proceso del desarrollo de la inteligencia» (Lapierre y Aucouturier 1985: 4).

Vigotsky (1989) halla una relación dinámica durante el desarrollo infantil entre acción y lenguaje en la que en un primer estadio domina la actividad y el lenguaje acompaña a la acción y en el siguiente el lenguaje precede a la acción funcionando como guía. Él la llamó «acción planificadora del lenguaje». Acción y lenguaje pues integran el mayor campo de aprendizaje del niño y aunque en los primeros años de su vida predomine el lenguaje dinámico, la lengua posteriormente dará las pautas. 
La escuela, encargada de educar a las personas facilitando su inserción social, debe mover en el niño las destrezas necesarias para hacerlo. (Gardner, 1987) afirma que desde los primeros años escolares es necesario facilitar al niño el acceso a distintos lenguajes, porque en este periodo maneja el lenguaje tanto para expresarse como para entender lo que le rodea, transitando por primera y a veces por última vez de forma natural entre los diversos lenguajes utilizando en cada momento el que mejor se adapta a sus necesidades expresivas. Si la escuela puede conseguir que a través de la acción-juego con lenguaje y pensamiento desarrolle aptitudes para aprender descubriendo (Bruner, 1984; Lapierre y Aucouturier, 1985), moverá su interés implicándolo en su propio aprendizaje. Facilitándole las herramientas (Vygotski, 2001) y andamiajes (Bruner, 1984) necesarios, le proporcionará satisfacción y reforzará sus estrategias.

\section{LAS SENDAS DEL LENGUAJE ESCRITO}

La escuela infantil dedica gran parte del tiempo lectivo a la enseñanza-aprendizaje de la lengua como instrumento imprescindible para expresarse, comunicarse y conocer, pero ha insistido tanto en la mecánica de la escritura, que como dice (Vygotski, 2001) se ha olvidado el lenguaje escrito como tal. Igualmente, ha desestimando la perspectiva social de su origen, la actuación del sujeto que lo su emplea para crear significados y dar sentido a la expresión y los conocimientos y el bagaje expresivo que acompañan al niño al acceder a la escuela, donde la acción educativa debe enriquecer los recursos que ya maneja cuando inicia la escolaridad (Zabalza, 1996).

La lengua escrita es parte de la expresión lingüística con componentes intelectuales, expresivos y motores que precisa: como actividad intelectual la configuración mental de estructuras lógicas; en el aspecto expresivo la creación de un andamiaje expresivo comunicativo, y en el aspecto motor desarrollar la motricidad gruesa y fina para dominar el cuerpo y el gesto (Lebrero, 2002). Su aprendizaje no se reduce como se creía a procesos perceptivos, visuales y motrices, sino que se fundamenta en el conocimiento metalingüístico (Arnaiz y Bolarin, 2016).

Los profesionales han intentado enseñar el lenguaje de forma eficaz utilizando distintos métodos, pero al no tratar adecuadamente sus aspectos expresivo-comunicativo y motriz, han ido apareciendo durante el proceso instructor arrastrando limitaciones cognitivas relacionadas con la comprensión y el sentido que llegan a dificultar la resolución de problemas (Rodríguez y Domínguez, 2016) o la legibilidad, impidiendo un aprendizaje efectivo.

Entre los métodos tradicionales se encuentran los llamados sintéticos (silábicos, alfabéticos, fónicos...), que parten de unidades lingüísticas simples (letras, sílabas) no significativas para alcanzar formas más complejas. Métodos analíticos (globales) que, se apoyados en el sincretismo y la percepción global infantil, utilizan unidades lingüísticas de estructura compleja, significativas para el niño (Ausubel, 2002) $\mathrm{y}$ acordes con sus motivaciones e intereses, para llegar al conocimiento de a las 
más simples. Los métodos eclécticos conjugan los dos anteriores considerando que en lengua se realiza un doble proceso de análisis-síntesis. Aunque son métodos diferentes, todos gestionan entrenar al niño en el código escrito, no desarrollar su competencia comunicativa.

Hacia mitad de 1960 la sociolingüística colocaba en el eje de la enseñanza de la lengua el concepto de competencia comunicativa, relacionando la teoría lingüística de Chomsky con factores socioculturales y originando nuevos enfoques como, lenguaje integral, lenguaje funcional y psicogénesis, que conciben la escritura como sistema de representación del lenguaje, no como transcripción fonética (Ferreiro y Teberosky, 1985), y valoran la riqueza expresiva que poseen los niños al ingresar en la escuela.

El lenguaje integral planteado por Kennet y Yetta Goodman (1990, 1992), con base en Vygotski, concibe el aprendizaje de lectura y escritura como actividades sociales que el niño aprende como el habla, interactuando con su entorno, manejando textos reales (cuentos, periódicos, anuncios...) y con un profesor-guía que elude la enseñanza directa de letras, palabras, etc., haciendo al niño dueño de su aprende movido por la necesidad de comunicarse. Las funciones lingüísticas El lenguaje funcional observa el contexto sociocultural y la función de la lengua escrita para comunicar significados. El enfoque representado por la psicogénesis de la escritura de Ferreiro y Teberosky (1985), con base piagetiana, utiliza distintas unidades escritas (palabras, frases, textos) partiendo de lo que el niño sabe para que aprenda el código alfabético, las diferencias existentes entre la lengua oral/escrita, las estructuras de los diferentes tipos de textos, su disposición gráfica y su vocabulario. Todos manejan textos múltiples desde el inicio del aprendizaje, consideran leer y escribir formas de comunicación y afirman que leer es buscar significado, no decodificar, proponiendo hacer «lectores plenos, no descifradores», (Ferreiro, 2002: 18).

Estos nuevos enfoques permiten subsanar la incomprensión y falta de sentido de los textos restaurando el valor expresivo y comunicativo del lenguaje, pero no su legibilidad. Ferreiro (2002: 58) incluso considera obsoleto debatir sobre los tipos de letra o los útiles para el aprendizaje de la escritura, alegando que «la escritura moderna es con las dos manos y con caracteres separados». En el contexto educativo de la escuela infantil incluidos estos enfoques, se sigue pasando de puntillas por el aspecto motriz para caminar como manifiesta (Calmy, 1977: 10) «demasiado aprisa hacia la abstracción verbal... sin haber buscado antes el apoyo de una integración motriz de la vivencia”, cuando en último término el pensamiento es acción interiorizada o transformada en una forma simbólica (Bruner, 1984). Esto no facilita que el niño adquiera un sistema gráfico ágil en su ejecución y fácilmente legible, es decir, maduro. Resta considerar que igual que la pantalla no sustituye al libro, el teclado nunca podrá sustituir a la escritura manual.

La manera de enfocar la actividad escritora y el punto de partida para llevarla a cabo tradicionalmente no ha variado, se sigue imponiendo la perspectiva visual y se aprenden las formas de escritura copiando modelos realizados por adultos cuyas con iconografías más o menos figurativas que imponen una estética concreta, 
obviando que el origen del gesto gráfico es motor (Lapierre y Aucouturier, 1985) y solo se hace gráfico cuando el niño lo estampa en un soporte, pero no cuando dibuja en el aire (Calmy, 1977). Imponer una estética especifica que anula la creatividad y desestima la utilización de la expresión artística como recurso didáctico que permite un aprendizaje significativo y motivador de diferentes contenidos en la etapa de Educación Infantil y copiar formas estáticas discrepa con la necesidad infantil de acción y con la actividad gráfica, "que es gestual y la gestualidad es trayectoria y por tanto dinamismo» (Calmy, 1977: 10).

Autores como Berenguer, Llamas y López (2016) afirman que en términos de innovación y cambio metodológico en Educación Infantil se han de relacionar otras variables, ellos apuestan por la creatividad y lateralidad como dos variables claves que se expresan en todos los lenguajes y formas de comunicación (verbal, plástica o escrita).

En investigaciones sobre el concepto de la creatividad motriz autores como Marchena, López y Ezquerro (2017) determinan que hay que prestar atención a la manifestación del movimiento y al lenguaje motor, pero también a todo el proceso que emplea el sujeto para expresarse. Por ello, consideran que es preciso tener en cuenta la distinción entre el proceso creativo motriz, el producto creativo y el producto creativo motriz.

Desde la experiencia en el aula surgió la necesidad de facilitar a los niños otra forma de enseñanza-aprendizaje de la escritura más adecuada a su naturaleza, a la dinámica de la propia actividad y a favorecer la parte emocional, pues sin ella no existe desarrollo armónico ni es posible acceder al pensamiento operativo.

La educación motriz vivenciada sitúa el desarrollo afectivo como centro y punto de partida de la acción psicomotriz primando la vivencia emocional, que genera una actitud vital y unos patrones de interacción cognitivos y sociales que se integran e influencian entre sí como base para construir la personalidad (Hernández, 2015)

Elegirla para educar el trazado y la escritura pareció el camino apropiado, porque considerada por Piaget imprescindible para construir pensamiento abstracto, permitía aprehender espacio, sentido, trayectoria y trazo de una forma dinámica y lúdica muy acorde con los intereses y la naturaleza infantil.

Este trabajo emprende ese camino, proponiendo sustituir la copia repetitiva de formas prefabricadas por una educación motriz vivenciada, global y unitaria utilizando el enfoque psicomotor. Se basa en desarrollar múltiples experiencias con el lenguaje dinámico corporal vivenciando los movimientos en el espacio para interiorizarlos antes de transponerlos al papel (Calmy, 1977), entender la relación movimiento-trazo, aprender la dirección y el sentido del trazado y la escritura occidental y hacerle ganar legibilidad.

\section{Metodología}

Esta investigación presenta la educación del trazado desde un punto de vista motriz apartándose del visual utilizado tradicionalmente. El auténtico valor de la 
actividad radica en el proceso motriz que impulsa el nacimiento del gesto gráfico (Calmy, 1977), no en obtener hojas con trazos, porque es en el proceso donde se producen los cambios, haciendo menos relevantes los resultados (Vigotsky, 2001) que, sin embargo, resultan concluyentes. Se compone de actividades motrices y plásticas porque es parte de un estudio más amplio que utiliza los lenguajes artísticos como armazón del aprendizaje.

El estudio empírico se ha apoyado en la educación vivenciada y el aprendizaje por descubrimiento a través de proyectos para estimular en los sujetos la negociación, la decisión conjunta y favorecer la colaboración, el respeto o la valoración del otro además del protagonismo activo. Es un método de múltiples conjeturas como afirma (Popper, 1980) donde se han facilitado a los sujetos las máximas posibilidades en actividades observables y materiales de diferente uso (Vigotsky, 1989). A la observación y la corrección de errores han seguido nuevos planteamientos de la investigación para poder cubrir los distintos enfoques que, como describe (Hilgers, 2013) se interpreta un material empírico en sintonía con la experiencia de la observación participante que consiste como define (Rodgers, 2001), en la participación activa del investigador en el proceso que se está estudiando.

La finalidad de este programa es conseguir que los alumnos, ejecutando y observando movimientos de las extremidades y desplazamientos del cuerpo, los vivencien y plasmen en el papel en trazos, configurando así los gestos gráficos que sustentan la escritura. De esta forma, comenzarán aprehendiendo el espacio en que se mueven y conociendo las posibilidades que tiene su propio cuerpo a la vez que prueban relaciones con objetos y compañeros. Posteriormente, irán asimilando la relación del movimiento con el trazo y la conversión del primero en grafismo, para finalmente traducir a la inversa.

\subsection{Objetivos Generales y Específicos}

Objetivo general

- Aprender el sentido del trazado y la escritura y realizar diferentes tipos de trazos.

\section{Objetivos específicos}

- Descubrir el espacio y los objetos respecto al cuerpo y las relaciones de este con los objetos y los otros.

- Conocer el propio cuerpo y sus posibilidades.

- Realizar movimientos y trayectorias y convertirlos en trazos.

- Analizar trazos diferentes para descubrir el movimiento.

- Realizar trazos que formen grafismos. 
4. MUestra

La muestra probabilística proporcionada por los profesores tutores de los grupos que han colaborado desde los diferentes centros abarca una población de 113 niños (67 niños y 46 niñas) de 5 años correspondientes a cinco centros escolares públicos, con poblaciones de medios socioculturales distintos; los datos han sido proporcionado por los tutores de cada grupo en los diferentes centros. Se han considerado el procedimiento ético y de confidencialidad de recogida de datos y los permisos pertinentes de las familias y los centros para la aplicación de los instrumentos.

GRÁFICO 1

Descripción de la población

\begin{tabular}{|c|c|c|}
\hline 30 & & \\
\hline 25 & & \\
\hline 20 & & \\
\hline 15 & & \\
\hline 10 & & \\
\hline 5 & & \\
\hline 0 & Hijos de familias extranjeras & Hijos de familias españolas \\
\hline CEIP A & 0 & 27 \\
\hline CEIP B & 21 & 0 \\
\hline CEIP C & 13 & 9 \\
\hline - CEIP D & 9 & 14 \\
\hline CEIP E & 13 & 7 \\
\hline
\end{tabular}

\subsection{Descripción sociológica de la muestra}

- Grupo A- compuesto por 27 sujetos (16 niños y 11 niñas) hijos de familias españolas con nivel sociocultural alto o medio alto.

- Grupo B- compuesto por 21 sujetos (13 niños y 8 niñas) hijos de familias inmigrantes con nivel sociocultural bajo

- Grupo C- compuesto por 22 sujetos (16 niños y 6 niñas) hijos de familias españolas e inmigrantes de nivel sociocultural medio a medio bajo.

- Grupo D- compuesto por 23 sujetos (10 niños y 13 niñas) hijos de familias españolas de nivel sociocultural medio alto e inmigrantes de bajo nivel sociocultural.

- Grupo E- compuesto por 20 sujetos (12 niños y 8 niñas) hijos de familias españolas de nivel sociocultural medio alto e inmigrantes de bajo nivel sociocultural. 


\subsection{Procedimiento}

Ejecutar un programa motriz-creativo realizando movimientos en el espacio y observando los movimientos de los otros para interiorizarlos, advertir las dificultades y una vez vivenciados reproducirlos gráficamente. Descifrar los grafismos representativos de movimientos y trayectorias y tornarlos en movimiento.

Acciones:

- Exploración dinámica del espacio para obtener un conocimiento sensorial y perceptivo del mismo que permita abstraer sus cualidades y aprehenderlo.

- Ejecución de movimientos dirigidos a adquirir conceptos básicos relacionados con el espacio y los objetos situados en él; perfeccionar el nivel gráfico, y facilitar el acceso a la escritura.

- Realización de ejercicios motrices precisando el sentido y la dirección del movimiento para orientar el gesto gráfico.

- Iniciación en la relación desplazamiento-trayectoria-trazado para aprehender el movimiento en el espacio y su conversión en escritura.

- Descifrado de los grafismos que representan trayectorias y su tradución en movimiento.

- Realización de intercambios de lenguajes para estimular oralidad, lectura y escritura poniendo título a dibujos, describiendo y escribiendo nombres de los elementos que contienen y narrando las historias originadas.

- El trabajo consta de 30 sesiones de una hora llevadas a cabo en horario lectivo.

\subsection{Instrumentos de evaluación}

Se han utilizado dos pruebas creadas para esta investigación, una cuantitativa y otra cualitativa. La primera mide el nivel gráfico de los alumnos, y la segunda es una entrevistar para los tutores.

\subsubsection{Prueba de madurez del grafismo}

Se necesitaba un instrumento que permitiera conocer el alfabeto gráfico (signos que forman la grafía personal) que cada sujeto posee y con el que se expresa con lenguaje propio en el dibujo espontáneo. Se creó para ello una prueba consistente en un sencillo relato descriptivo de unos minutos incluyendo elementos que necesitan para su realización emplear los garabatos básicos de la escritura (como el bucle para dibujar árboles o rizos). Se hace la narración pidiendo a los sujetos que la dibujen mientras escuchan, indicándoles que solo pueden «dibujar» pero no "Colorear" para que se limiten a trabajar con líneas, no pierdan ningún elemento descrito y utilicen en el grafismo espontáneo los signos que tienen asimilados. 
La prueba también permite averiguar los conocimientos en lectoescritura, al pedir a los sujetos que pongan nombre a elementos incluidos en sus dibujos para saber si han iniciado la comprensión del código escrito, averiguar si conocen la grafía de alguna palabra y detectar el tipo de letra (cursiva/script) que utilizan espontáneamente.

La prueba atiende a conocer los signos gráficos empleados trazando espontáneamente desde la narración, la utilización espontanea de letra cursiva y el conocimiento de palabras escritas.

Se valora con puntuación directa, contando el número de garabatos básicos distintos realizados por cada sujeto, el número de palabras conocidas si las escriben, y el tipo de letra utilizada. La prueba se ha implementado al principio y al final de la investigación.

\section{Relato descriptivo}

En un hermoso prado donde la hierba crecía muy recta formando filas había un castillo con su torre, y junto a él un árbol con el tronco grueso y la copa muy rizada. El árbol servía de escondrijo a dos serpientes perezosas que se enrollaban a su tronco o caminaban cerca de él intentando cazar ratoncillos y otros animalitos que les servían de alimento.

El prado era un lugar luminoso en el que brillaba el sol aunque asomara alguna nube derramando sus gotas de lluvia, precisamente por eso estaba siempre lleno de flores de todos los colores. Muy lejos, en el fondo, había una larga fila de montañas de pico que cerraba el prado a los curiosos.

Se dice que allí viven duendecillos mágicos que tienen el poder de hacerse invisibles, por eso unas veces se ven y otras no. ¿Se ve alguno en tu dibujo?

\section{Relato descriptivo: cuento}

Otra fuente utilizada ha sido la observación participativa, importantísima a lo largo de toda la actividad como la forma más eficaz de conocer paso a paso la marcha del proceso con niños pequeños, proporcionando datos cualitativos que los empíricos no podían facilitar y que, como mantiene (Vigostky, 1989: 36) «si se extraen objetivamente» con rigor científico, dichas observaciones adquieren estatus de hechos perfectamente válidos. Esta afirmación concede valor a la observación en la labor investigadora, que en este trabajo ha sido fundamental y rigurosa, controlando con una rúbricas si realizaba cada sujeto movimientos y trayectorias, y si traducía los movimientos a trazado y el trazado a movimiento y señalando las dificultades que encontraba. 


\section{Entrevista a profesores colaboradores}

1. Exprese su opinión sobre el programa para educar el grafismo aplicado a sus alumnos.

2. ¿Lo encuentra adecuado a la edad de los niños?

3. Comente el resultado de haber insertado esta experiencia en el trabajo habitual de clase.

4. ¿Le parecen de mayor interés los ejercicios motrices, los gráficos o ambos?

5. ¿Qué opinión le merece el programa en relación con la adquisición de competencias (lingüística, artística, social, de resolución de problemas, colaborativa)?

6. Diga si ha percibido que el programa da protagonismo al niño en su aprendizaje y si le induce a asociar y relacionar cosas nuevas.

7. ¿Ha observado si sus alumnos, después de participar en el programa, han progresado respecto a la constancia para concluir los trabajos, solucionar dificultades y reducir la ansiedad ante los fallos?

8. ¿Cree que la participación en este programa ha estimulado en sus alumnos la iniciativa personal y la autonomía?

9. ¿Le parece que las actividades llevadas a cabo favorecen la valoración del trabajo propio/de otros y el interés por escribir?

10. ¿Encuentra positivo incluir en su trabajo docente este tipo de actividades?

\subsubsection{Entrevista a tutores}

Para obtener una información más amplia se ha preparado una entrevista para los tutores con 10 preguntas, 5 abiertas y 5 cerradas, para conocer su opinión sobre el programa en general, su adecuación a la edad de los niños, la facilidad de inserción en horario escolar y otros datos relacionados con la adquisición de competencias, el desarrollo de asociaciones y relaciones nuevas o el aprendizaje por descubrimiento, así como su validez para impulsar la iniciativa y la autonomía o su contribución a la valoración del trabajo y al interés por la expresión escrita.

\subsection{Descripción del programa}

El programa incluye ejercicios motrices generales, ejercicios gráficos y juegos gráficos. Los primeros son movimientos libres de exploración del espacio con puntos de referencia y accesorios para utilizar libremente. Los ejercicios gráficos valen para estructurar el gesto, orientándolo en un espacio concreto, en la dirección y sentido de la escritura occidental y hacerlo rítmico. Los juegos gráficos tienen un 
sentido más lúdico-artístico, pero también toman parte en el perfeccionamiento del trazo.

\subsubsection{Ejercicios generales}

Su objeto es explorar el espacio realizando movimientos motrices libres vivenciados y observados, movimientos segmentarios y recorridos con o sin obstáculos y finalmente su análisis perceptivo para que los sujetos analicen sus vivencias personales y las de los otros.

FiguRA 2

Contenido de los ejercicios generales para el análisis perceptivo

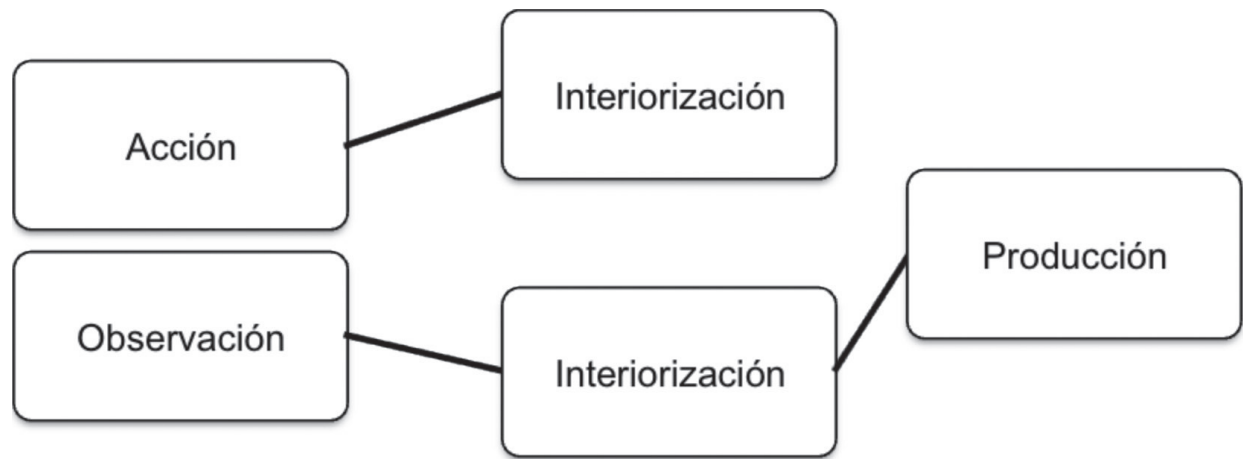

Al comenzar la actividad se proporcionaron a los sujetos objetos de motricidad música y plástica para que jugaran libremente. Una vez conocidos, todos utilizaron un mismo objeto para descubrir que se podía hacer con él, después varios. Primero trabajaron el gesto, coger, lanzar..., luego sin objetos, gesticulando. Después el сuerpo. ¿Qué podemos hacer? Saltar, estirarnos... también con ritmos. Finalmente realizaban un análisis perceptivo de la actividad.

\subsubsection{Ejercicios gráficos}

Son actividades motrices con reglas concretas para potenciar las direcciones prioritarias de la escritura occidental en las que los sujetos investigados se turnaban como actores/observadores llevando a cabo los movimientos (acción) o mirando (observación) y realizando el trazado simultáneo a los movimientos (producción). Actores y observadores interiorizaban los movimientos y los observadores, además, trazaban. 
FIGURA 3

Funciones de distintos sujetos

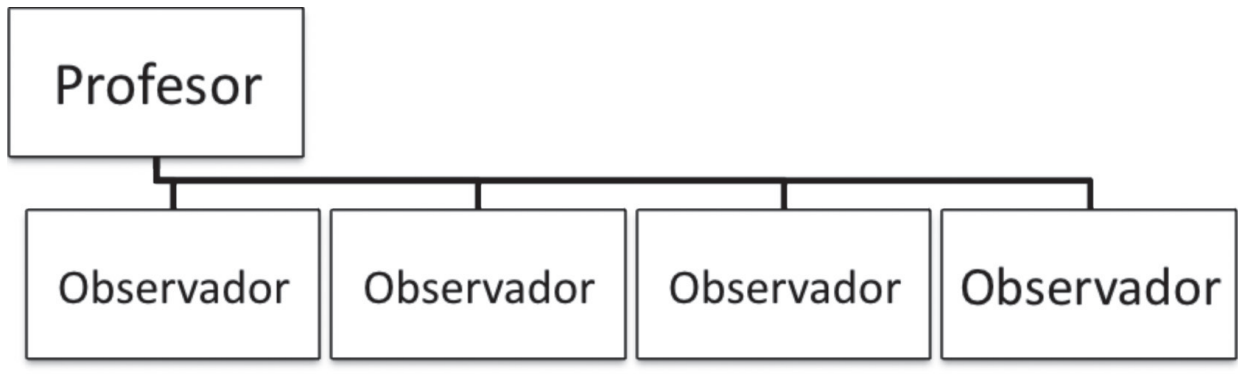

\section{Actor}

Pautas para realizar los ejercicios gráficos:

- Para que el sentido izquierda-derecha fuera igual para todos, el actor estaba situado frente al grupo de observadores dándoles la espalda. El grupo de observadores se colocaba alineado frente a él y la educadora detrás del grupo para controlar la acción.

- Los observadores, de pie o sentados, tenían material para trazar mientras el actor realizaba movimientos o desplazamientos, "caminos".

- Se empezó trazando sobre soporte amplio (papel continuo sobre pared o suelo, arena, etc.) reduciéndolo hasta el folio, utilizando palos sobre la arena y rotuladores o ceras para conseguir un trazo continuo sobre papel.

- En los ejercicios gráficos se ha potenciado la dirección de la escritura occidental: de abajo arriba, de arriba abajo y de izquierda a derecha. Comenzando siempre desde la izquierda y eludiendo el sentido de derecha-izquierda.

- Los cuatro trazados que se incluyen (Imagen 1) representan recorridos, en ellos el punto indica el lugar de inicio.

- El trazo vertical corresponde al movimiento de avanzar y retroceder. Un palo sobre arena deja la huella detrás al avanzar y delante al retroceder.

- Para que los sujetos relacionaran los conceptos arriba y abajo con el movimiento, se les pidió dibujar verticales de cerca a lejos de sí y al contrario sobre papel continuo, sosteniéndolo en vertical y volviéndolo a posar en el suelo explicándoles -arriba es lejos de ti y abajo cerca-.

- Para saber si reconocían distintas trayectorias se les daban hojas con ejercicios como el de la Imagen 1 para que realizaran los recorridos trazados. 

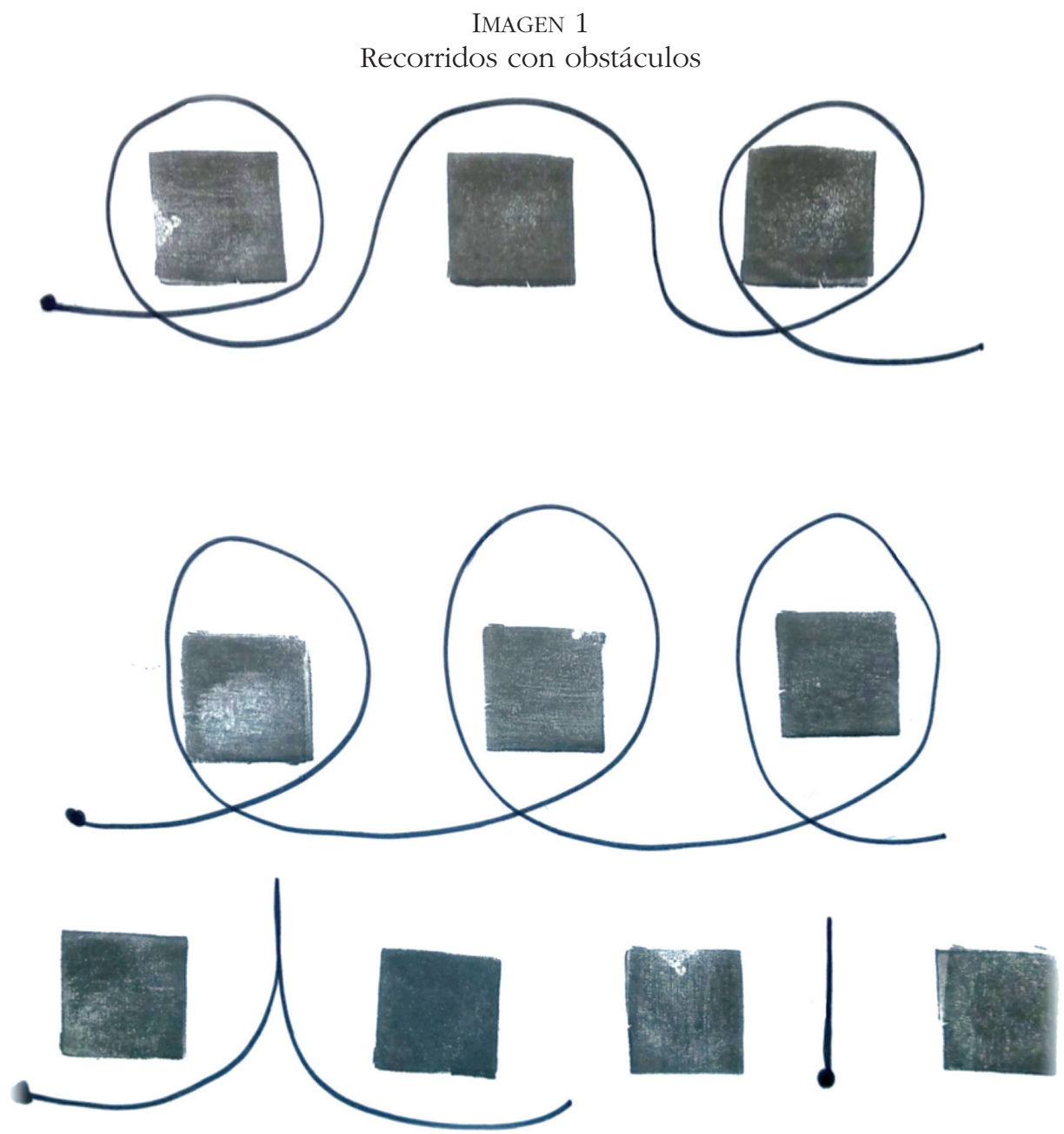

Juegos gráficos. Son juegos con consignas, realizados con materiales diversos (pegatinas, elementos para encolar y rotuladores gruesos y finos) practicados individualmente y en grupo a partir de algún trazo realizado por el niño. Potencian la atención, la creatividad, la perfección en el trazado, el conocimiento del espacio y el trabajo en equipo. En los juegos gráficos, aunque se ha dado alguna consigna, se ha dejado el resto del trabajo abierto a la creación personal. 
IMAGEN 2

Juegos gráficos grupales. (Trabajo de alumno)

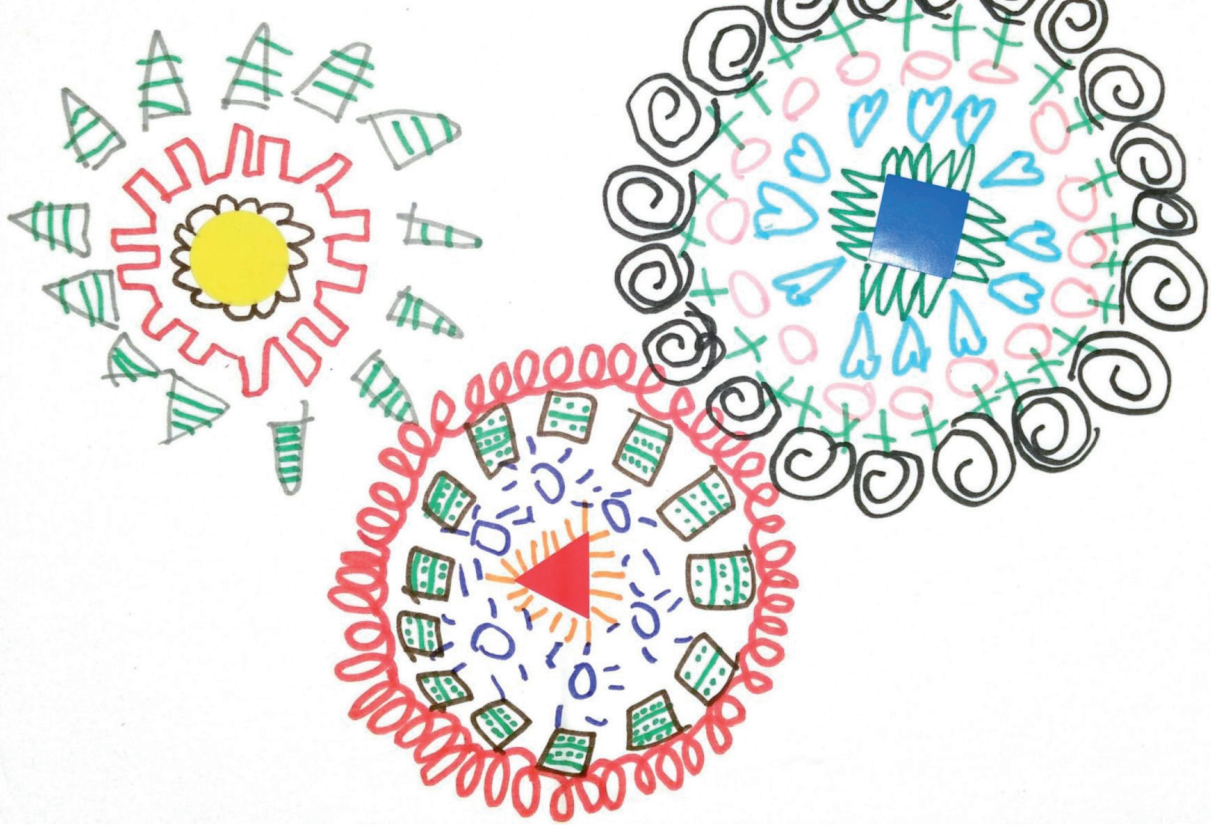

\section{RESUlTADOS}

\subsection{Resultados de la prueba de grafismo}

\subsubsection{Trazado de garabatos}

Pre-prueba. Grupos A, B, C, D y E. El 45,13\% de sujetos trazaron todos los garabatos básicos previos a la escritura; el 0,88\% no trazaban bucles, zigzags, curvas ni ondas; el 9,73 no trazaban bucles, zigzags ni curvas; el 20,35\% no trazaron bucles ni curvas, y el 23,89\% fallaron el bucle.

Post-prueba. Grupos A, B, C, D y E. El 79,64\% de sujetos trazaron todos los garabatos básicos previos a la escritura, el 0,88\% no traza bucles, zigzag ni curvas, el 2,65\% no trazaban bucles, zigzag ni ondas, un 9,73 no trazaron bucles y ondas y un 7,07 no trazaron bucles.

Los resultados obtenidos muestran que aumentaron en 34,51\% los sujetos que después de realizar el programa trazaban todos los garabatos básicos del alfabeto gráfico espontáneamente, disminuyendo en un 34,52\% los que solo trazan algunos. 
GRÁFICO 2

Prueba pre/postprograma de nivel gráfico de los estudiantes

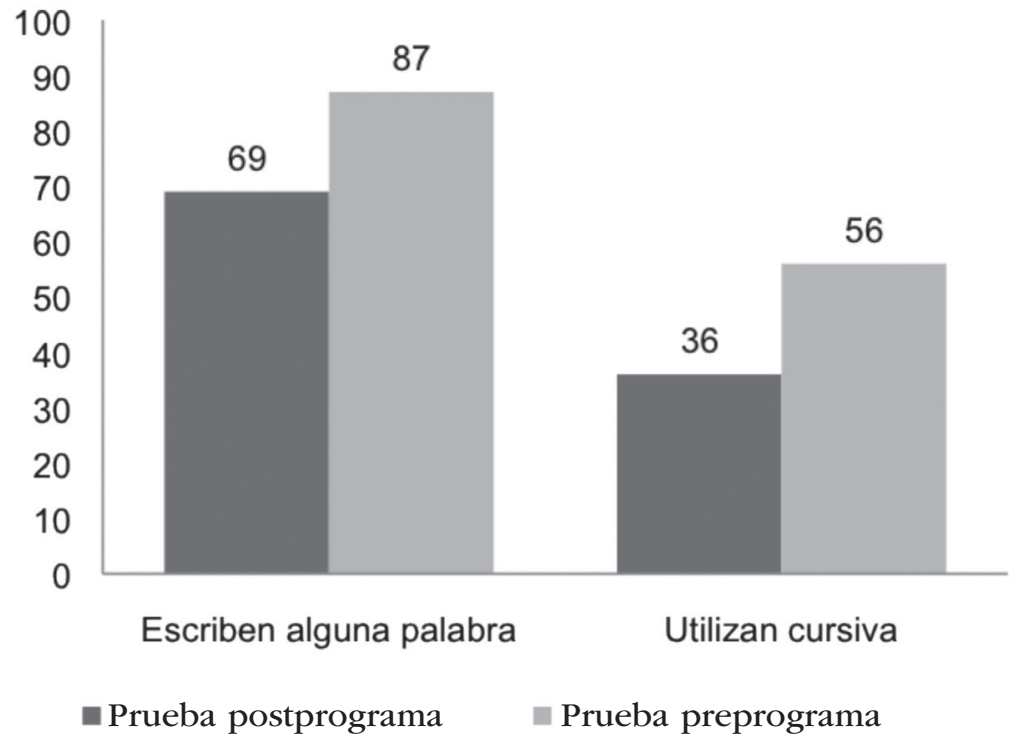

\subsubsection{Utilización de letra cursiva}

Al comenzar el programa 36 sujetos utilizaban de forma espontánea la letra cursiva y 56 al finalizar. También al comenzar el programa 69 sujetos escribían algunas palabras y 87 al acabarlo.

Los resultados muestran que en la postprueba ha aumentado el número de sujetos que utilizan letra cursiva y escriben algunas palabras.

\subsection{Resultado de la observación}

La inserción del trabajo en el aula ha resultado positiva para el resto de actividades, como han señalado los tutores. Las actividades cooperativas han favorecido el intercambio personal y el trabajo en grupo (Cooper, 2009; Johnson y Johnson, 2014) frente a la tendencia al individualismo en esta edad, impulsando la colaboración y contribuyendo a valorar el trabajo personal y de los otros. Los sujetos se han ayudado de la oralidad para solucionar las actividades más difíciles, confirmando que utilizaban el lenguaje oral para planificar (Vigotsky, 1989) y ante las dificultades para trazar o traducir trazado a movimiento buscaban el "andamiaje» (Vigotsky, 2001; Bruner, 1984) de la responsable del programa o de algunos compañeros cuya mediación les permitía mejorar sus capacidades (Vigotsky, 1981). 
Se ha verificado que la práctica del programa ha estimulado a los sujetos a relacionarse con los compañeros, ayudarse mutuamente y crecer en sociabilidad. Han intervenido activamente, (Zabalza, 1987; Piaget, 1965; Vigotsky, 2001; Bruner, 1984) decidiendo, opinando y valorando, ganado así autonomía y confianza.

El juego con el espacio y el trazado ha despertado el interés de sujetos que se mostraban resistentes a escribir y ha enfocando a todo el grupo a expresarse de forma creativa, transitando entre distintos lenguajes (Gardner, 1987).

\subsection{Resultado de la entrevista a profesores}

- Valoración del programa. Los tutores A, C y E lo encuentran «creativo o muy creativo"; A, "práctico»; B, «enriquecedor y fácil de realizar»; D, "novedoso". A, D y E dicen que "harían estas actividades un día a la semana». B y C, que "estimula a los niños».

- Adecuación a la edad. Todos los tutores lo encuentran adecuado a la edad.

- Encaje en la actividad escolar. B, valora "haber elegido la hora apropiada" C y E afirman que «rompe la rutina»; C, que «es motivador», y E que «facilita los aprendizajes".

- Ejercicios de mayor interés. A los tutores les parecen interesantes ambos tipos. B y C, encuentran «más completos los ejercicios gráficos». D, opina que «igualmente, los dos dinamizan el aprendizaje» y E, dice que «son complementarios".

- Valor competencial. Los tutores valoran que el programa estimule la oralidad y la escritura. A y B afirman que, «incentiva a resolver problemas». A, B y C dicen que "favorece la creatividad». A, D y E opinan que "anima a la colaboración» y C y E creen que «estimula las relaciones entre los niños».

- Contribución al protagonismo del niño, a buscar asociaciones y relaciones nuevas. Todos los tutores opinan que son actividades que los hacen aprender descubriendo por sí mismos. B, valora que «descubren desde el movimiento" y E, que "aprenden a asociar y relacionar para entender la correspondencia movimiento-trazo".

- Desarrollo de constancia, seguridad, autonomía e iniciativa. Todos los tutores opinan que impulsa los valores referidos. D afirma que «hace más seguros a niños con dificultades». A, que "pierden el miedo a equivocarse». C, que «aceptan mejor los fallos». E, que "Son más constantes y no les importa repetir».

- Valoración del trabajo e interés por la expresión escrita. Todos los tutores afirman que el programa estimula a valorar los trabajos propios y de otros y el interés por trazar y escribir. B dice «quieren hacerlo bien y preguntan cuando dudan». C, "fuera de las sesiones intentan trazar y escribir por iniciativa propia». E, "Son más críticos con sus trabajos».

- Opinión sobre la inclusión de este estilo de educar el grafismo en su clase. 
Todos los tutores están interesados en adoptar la educación motriz vivenciada. A, B y D ya lo están haciendo

\section{CONCLUSiOnes}

Los alumnos y profesores han encontrado estimulante participar en este trabajo, cuyos resultados confirman que las actividades realizadas han dinamizado el proceso lectoescritor. En primer lugar, porque estimulaba a los sujetos a trabajar incluso en labores rutinarias y también porque los sujetos se han sentido protagonistas, responsables de su trabajo y coautores de una obra común.

El proceso les llevaba a explicar cómo iban a realizar un desplazamiento traduciendo el trazado o transcribiendo movimientos a trazos, lo que ha ayudado a los más tímidos a abrirse a la comunicación y a todos a ganar en oralidad.

La práctica del programa ha estimulado la relación entre compañeros, la ayuda mutua y la sociabilidad, prestando los más hábiles ayuda a otros y siendo muy satisfactorio para todo el grupo, porque todos los sujetos han intervenido activamente, en la línea de investigaciones como las de Johnson y Johnson (2014).

El programa ha hecho alumnos lectores como demandaba Ferreiro (2002) aprendiendo el código alfabético y las diferencias entre lengua oral y escrita, pudiendo estructurar diferentes tipos de texto, su disposición gráfica y vocabulario. Así han utilizado frases más largas y mejor construidas y aumentado su interés por utilizar la escritura.

Respecto al objetivo de aprender el sentido del trazado y la escritura y realizar diferentes tipos de trazos, los sujetos han desarrollado destreza técnica, agilidad, seguridad y perfección en el trazo.

Las dificultades han estimulado a los sujetos a ser críticos y perseverantes, haciendo emerger mecanismos frente a la frustración.

La observación ha permitido comprobar que los sujetos usaban nuevos recursos para descubrir el movimiento y transformarlo en grafismo, para comparar y descifrar trazados estableciendo poco a poco estrategias propias y buscando recursos para conseguirlo. Los alumnos han ido descubriendo el espacio y los objetos respecto al cuerpo y las relaciones de este con los objetos y los otros. Es decir investigaban, crecían en autonomía y aumentaba su interés conociendo el propio cuerpo y sus posibilidades. También los profesores con este proceso salen beneficiados porque la inserción del trabajo en el aula ha resultado positiva para el resto de actividades. Lo que concuerda con lo recogido en la entrevista sobre el programa. El profesorado considera que es un programa creativo, práctico, enriquecedor y fácil de realizar. Los profesores consideran que es innovador y estimulador al dar protagonismo al niño. Entre otros aspectos destacan que la utilización de una metodología activa y vivenciada ayuda a los alumnos a tener iniciativa, tomar decisiones y resolver problemas, proporcionando mayor seguridad y autonomía en el aprendizaje. 
MARÍA LUISA BENÍTEZ SÁNCHEZ Y CRISTINA SÁNCHEZ ROMERO

PROCESOS EDUCATIVOS EN EDUCACIÓN INFANTIL MEDIANTE EL GRAFISMO Y LA ESCRITURA

Se puede afirmar en la línea argumentada de Calmy (1977) que educar el gesto gráfico desde el movimiento motriz es una alternativa poderosa en Educación Infantil porque: muestra su validez para resolver las dificultades que plantea el aprendizaje de la escritura; es más lúdico, dinámico y creativo que el sistema tradicional y el niño al ser protagonista lo considera propio. Finalmente, se evidencia que promueve la ayuda, la autoestima, y contribuye a la adquisición de competencias para aprender a aprender.

\section{REFERENCIAS BIBLIOGRÁFICAS}

Arnaiz, P. y Bolarín, M. ${ }^{a}$ J. (2016). Introducción a la psicomotricidad. Madrid: Editorial Síntesis S. A.

Antón Sancho, A. y Gómez Alonso, M. (2016). La geometría a través del arte en Educación Infantil. Enseñanza \& Teaching: Revista Interuniversitaria de Didáctica, 34, (1), 93-117. doi: http://dx.doi.org/10.14201/et201634193117.

Arnheim, R. (1976). El pensamiento visual. Barcelona: Editorial Paidós Ibérica.

Arnheim, R. (1989). Nuevos ensayos sobre psicología del arte. Madrid: Alianza Editorial S. A.

Ausubel, D. (2002). Adquisición y retención del conocimiento: una perspectiva cognitiva. Barcelona: Ediciones Paidós Ibérica.

Berenguer Sánchez, R.; Llamas Salguero, F. y López Fernández, V. (2016). Relación entre creatividad y lateralidad en Educación Infantil. Enseñanza \& Teaching: Revista Interuniversitaria de Didáctica. 34, (2) 65-75. https://doi.org/10.14201/et20163426575.

Bruner, J. (1984). Acción, pensamiento y lenguaje. Madrid: Alianza Editorial.

Calmy, G. (1977). La educación del gesto gráfico. Barcelona: Editorial Fontanella.

Cooper, J. (2009). Aprendizaje Colaborativo. Vicerrectoría Académica, Instituto Tecnológico $y$ de Estudios Superiores de Monterrey, 37. Retrieved from http://www.sistema.itesm. $\mathrm{mx} / \mathrm{va} /$ dide/publicaciones/inf-doc/Colaborativo.PDF.

Ferreiro, E. (2002a). Pasado y presente de los verbos leer y escribir. México: Ed. Fondo de Cultura Económica.

Ferreiro, E. (2002b). Relaciones de (in) dependencia entre oralidad y escritura (pp. 151-172). Barcelona: Editorial Gedisa.

Ferreiro, E. y Teberosky, A. (1985). Los sistemas de escritura en el desarrollo del niño. España: Siglo XXI editores.

Gardner, H. (1987). Arte, mente y cerebro-una aproximación cognitiva la creatividad. Barcelona: Paidós Ibérica.

Gardner, H. (1995). Mentes creativas. Barcelona: Ediciones Paidós.

Goodman, K. (1990). El lenguaje integral: un camino fácil para el desarrollo del lenguaje. Rev. Lectura y Vida, año 11, n. ${ }^{\circ} 2$.

Goodman, Y. (1992). Las raíces de la alfabetización. Rev. Infancia y Aprendizaje, n. ${ }^{\circ}$ 58, $29-42$.

Hernández, A. (2015). Guía de actuación y evaluación en psicomotricidad vivenciada. Madrid: CEPE.

Hilgers, M. (2013). Observation participante et comparaison. Anthropologie et Sociétés, 37 (1), 97. https://doi.org/10.7202/1016149ar.

Johnson, D. W. y Johnson, R. T. (2014). Aprendizaje cooperativo en el siglo XxI. Anales de Psicologia. https://doi.org/10.6018/analesps.30.3.201241 
Lapierre, A. y Aucouturier, B. (1977). Educación vivenciada. Barcelona: Editorial Científico-Médica.

Lapierre, A. y Aucouturier, B. (1985). Los contrastes. Barcelona: Editorial Científico-Médica.

Lebrero, M. P. y Lebrero, M. T. (2002). Lectura y escritura en los primeros años: enseñanza-aprendizaje. Madrid: Educación Permanente UNED.

Marchena Cruz, P.; López Fernández, V. y Ezquerro Cordón, A. (2017). Un estudio exploratorio de la relación entre la inteligencia musical, viso-espacial, corporal-cinestésica y creatividad motriz en el proceso de enseñanza-aprendizaje. Enseñanza \& Teaching: Revista Interuniversitaria de Didáctica. https://doi.org/http://dx.doi.org/10.14201/ et20173525575.

Marín Ibáñez, R. (1984). La creatividad y la educación plástica. II Seminario de Artes Plásticas.

Piaget, J. (1967). Seis estudios de psicología. Barcelona: Editorial Planeta Agostini.

Piaget, J. (1985). Seis estudios de psicología. Barcelona: Editorial Planeta Agostini.

Rodgers, D. (2001). Haciendo del peligro una vocación: Antropología, violencia, y los dilemas de la observación participante. Programa de Estados En Crisis. Documentos de Trabajo.

Rodríguez Hernández, M. ${ }^{a}$ M. y Domínguez Fernández, J. (2016) Dificultades del lenguaje que influyen en la resolución de problemas. Enseñanza \& Teaching: Revista Interuniversitaria de Didáctica, 34 (2), 17-42.

Teberosky, A. (2000). Los sistemas de escritura. Congreso Mundial de Lecto-escritura. Valencia.

Vygotski, L. S. (1979). El desarrollo de las funciones psíquicas superiores. Barcelona: Editorial Crítica.

Vygotski, L. S. (1989). El desarrollo de los procesos psicológicos superiores. Barcelona: Editorial Crítica.

Vygotski, L. S. (2001). Pensamiento y lenguaje. Madrid: Antonio Machado Libros.

Zabalza, M. A. (1987). Didáctica de la Educación Infantil. Madrid: Editorial Narcea.

Zabalza, M. A. (Coord.) (1996). Calidad en la Educación Infantil. Madrid: Editorial Narcea. 